\title{
RESEARCH
}

Open Access

\section{Health-related quality of life in a group of Egyptian children and adolescents with type 1 diabetes: relationship to microvascular complications}

Zeinab Mohammed Monir ${ }^{1}$, Mona Hussein El Samahy², Ehab Mohammed Eid ${ }^{3}$, Abla Galal Khalifa', Soheir abd-El Mawgood abd-ElMaksoud ${ }^{4}$, Mohamed Abdel Moneim Abbas ${ }^{1}$ and Hend Helmy Abd El Ghaffar ${ }^{1 *}$ (i)

\begin{abstract}
Background: The increased prevalence of type 1 diabetes mellitus in children and adolescents with its complications, especially microvascular ones (retinopathy, nephropathy, and neuropathy) that affect the expectancy of their lives, besides imposing restrictions on their physical, emotional, and social functioning, adversely affecting their quality of life, in turn would lead to worsening of their compliance and adherence to the treatment with subsequent hazards on metabolic control, development, and progression of adverse diabetic complications that might cause multiple organ damage and impose more disease burden and impact the quality of life of the growing young diabetics and their families.
\end{abstract}

Aim: The aim of the present study was to assess health-related quality of life of a group of Egyptian children and adolescents with type 1 diabetes in addition to investigate the relation of microvascular complications and other sociodemographic and clinical indicators to their quality of life.

Subjects and methods: This case-control study was executed in the Diabetes Clinic and in the outpatient clinics, Children's Hospital, Ain Shams University, and Medical Research Centre of Excellence clinics, June 2013-June 2015, which was carried out on 60 children and adolescents with type 1 diabetes mellitus, with ages ranged between (818) years compared with 60 apparently healthy children matched as regards their age, sex, and socioeconomic status.

Results: No significant differences between studied diabetic and healthy children (8-12 years) (total generic healthrelated quality of life score mean $=(77.05 \pm 14.58$ vs. $79.32 \pm 11.15$, respectively). But there was a significant decrease for studied diabetic adolescents (13-18 years) compared to healthy peers (64.37 \pm 14.54 vs. $74.74 \pm 13.34$, respectively). Microvascular complications impacted the health-related quality of life of type 1 diabetic children and adolescents, the most worsen effect was associated with neuropathy $(p<0.001)$. Statistically significant negative correlations were found between health-related quality of life domains with all metabolic control parameters, and the strongest correlation was between treatment adherence scale with $\operatorname{HbA1c} \%\left(r=-0.941, p^{<} 0.001\right)$.

Conclusion: Health-related quality of life of the studied diabetic children and adolescents was negatively affected by the development of microvascular complications, especially diabetic neuropathy, while positively affected by achieving good metabolic control (HbA1c $<8 \%)$.

Keywords: Diabetes mellitus type 1, Microvascular complications, Health-related quality of life

\footnotetext{
*Correspondence: hendhelmy_nrc@yahoo.com

'Department of Child Health, National Research Centre, Cairo, Egypt

Full list of author information is available at the end of the article
} 


\section{Background}

Type 1 diabetes mellitus (T1DM) is one of the most common pediatric chronic diseases, in which a progressive autoimmune $\mathrm{T}$ cell-mediated $\beta$ cell destruction of pancreatic islets in genetically susceptible individuals (Wang et al. 2017), resulting in loss of insulin secretion (Larsson et al. 2016). Numerous studies have reported an increasing incidence of type 1 diabetes in children worldwide (Patterson et al. 2015; El Wakeel et al. 2017; Sabry et al. 2018). It has major health consequences for individuals and societies. Management of type I diabetes mellitus (T1DM) is lifelong and challenging, considered an overwhelming demanding disease that affects all aspects of patient's life and thus can negatively affect the mental and physical health of the diseased children, impacting their health-related quality of life (HRQOL) (Jaser et al. 2017).

Several studies have demonstrated that diabetes has a strong negative impact on HRQOL, relative to the general population (Abuawad 2013; Jain et al. 2014; Nielsen et al. 2016), especially in the presence of complications (Hirai et al. 2013; Smith-Palmer et al. 2016). Diabetic neuropathy greatly reduces the quality of life in people with DM through increased disability and assuming more hospitalizations than other diabetic complications (Mahmood et al. 2009; Tesfaye and Selvarajah 2012; Abuawad 2013). Furthermore, greater pain levels in patients suffering distal symmetric polyneuropathy (DSPN), corresponded with higher levels of emotional distress, more sleep problems, and lower ratings of functioning and HRQOL (Vileikyte et al. 2017). The negative impact of severe hypoglycemic episodes on the QOL of young people was demonstrated in many studies, due to increased fear and anxiety, reduced productivity, and increased healthcare costs. Fear of hypoglycemia may promote compensatory behaviors in order to avoid hypoglycemia, such as decreased insulin doses, resulting in poor glycemic control, and an increased risk of serious health consequences, with further contribution to the negative impact on health and QOL (Hoey et al. 2001; Nordfeldt and Ludvigsson 2005; Fidler et al. 2011; Bahia et al. 2017).

There is a growing interest in the study of HRQOL, which has become an important end-point measure from the clinical and epidemiological point of view ( $\mathrm{Ra}$ vens-Sieberer et al. 2006; Floyd et al. 2017), commonly used as an outcome indicator because patient cooperation forms the core of health plans for incurable diseases (Lontchi-Yimagou et al. 2017).

It has been shown that poor HRQOL in patients with diabetes is associated with adverse outcomes and increased mortality (Kleefstra et al. 2008; de Souza et al. 2015). Enhancing QOL and well-being in people with diabetes is as important as metabolic control and prevention of secondary morbidity (Caferoğlu et al. 2016).

\section{Methods}

The aim of the present study was to assess health-related quality of life of a group of Egyptian children and adolescents with type 1 diabetes and to investigate the relation of microvascular complications and other sociodemographic and clinical indicators to their quality of life.

\section{Participants}

This case-control study included 60 children and adolescents with type 1 diabetes mellitus (84 type 1 diabetic patients recruited from the regular attendants of the Diabetes Clinic, Children's Hospital, Ain Shams University and Medical Research Centre of Excellence clinics, who fulfilled the inclusion criteria and accepted to participate in the study, 24 patients dropped out through the study and 60 patients completed the study). Written informed consent was obtained from the parents after explanation of the aim of the study, its benefits and expected risks for their children if they participate in the study and informed verbal assent was taken also from all participating children after a simplified explanation of the aim and benefits of the study for them. They were $22(36.7 \%)$ males and 38 (63.3\%) females, with age range (8-18 years), with a mean of $13.03 \pm 2.78$ years. "The involved diabetic children fulfilled the inclusion criteria of age (8-18 years), both sexes of cases diagnosed with Type 1 diabetes mellitus who are receiving insulin therapy and on regular visits to diabetes clinic, excluding cases who are associated with another chronic disease (e.g. chronic renal failure, cardiac diseases chronic chest disease ... etc.) and co-morbid psychiatric disorders (e.g. major depressive disorder, autistic disorder ... etc.), that may affect quality of life".

A control group of 60 healthy children and adolescents (recruited from the outpatient clinics and relatives of the diabetic children), with no chronic diseases or obvious medical disorders and not receiving any medications were matched for age, sex, and socioeconomic levels with the study diabetic group. They were 23 (38.3\%) males and 37 (61.7\%) females, with age range (AlvaradoMartel et al. 2015; American Diabetes Association 2018; Anderson et al. 2017; Arabiat and Al Jabery 2013; Bahia et al. 2017; Bai et al. 2017; Barcellos et al. 2014; Baş et al. 2011; Bisegger et al. 2005; Caferoğlu et al. 2016; Chawla 2004 ) years with a mean of $12.43 \pm 2.66$ years.

According to the presence of microvascular complications, patients were subdivided into two groups; Group I included 30 uncomplicated children and adolescents with T1DM, with age range (Alvarado-Martel et al. 2015; American Diabetes Association 2018; Anderson et al. 2017; Arabiat and Al Jabery 2013; Bahia et al. 2017; 
Bai et al. 2017; Barcellos et al. 2014; Baş et al. 2011) years with a mean of $11.25 \pm 1.92$ years. They were 12 (40.0\%) males and 18 (60.0\%) females. Group II included 30 children and adolescents with T1DM, and they had one or more diabetic complications, with age range (Arabiat and Al Jabery 2013; Bahia et al. 2017; Bai et al. 2017; Barcellos et al. 2014; Baş et al. 2011; Bisegger et al. 2005; Caferoğlu et al. 2016; Chawla 2004) years with a mean of $14.80 \pm 2.35$ years. They were $10(33.3 \%)$ males and $20(66.7 \%)$ females. According to age, patients and controls were subdivided into two groups (to correspond to the classification of age of PedsQL $\left.{ }^{\mathrm{mm}}\right)$.

\section{Processes}

All patients and controls were subjected to the following:

I. Full history taking: laying stress upon:

A. Sociodemographic data: (for all patients and controls) name, age, sex, and socioeconomic class.

B. Medical history of diabetes: (for cases)

1) Age of onset and duration of the disease and insulin therapy.

2) History suggestive of acute metabolic complications (in the last year prior to the study); frequency of hypoglycemia or ketoacidosis and number of hospital admission due to severe attacks of hypoglycemia or diabetic ketoacidosis.

3) History suggestive of chronic diabetic complications: ocular manifestations suggestive of diabetic retinopathy (persistent blurring of vision or flashes of light), peripheral neuropathy manifestations (tingling, numbness, paresthesia, impaired or lost sensation), renal manifestations suggestive of diabetic nephropathy (polyuria, oliguria, dysuria, loin pain, or haematuria).

II. Full clinical examination: Thorough clinical examination was done with particular emphasis on:

A. Routine examinations for all patients and controls:

1. Auxological assessment; growth was assessed through auxological measurements of weight $(\mathrm{kg})$ and height $(\mathrm{cm})$, body mass index $\left(\mathrm{BMI}=\right.$ weight in $\mathrm{kg} /$ height in $\left.\mathrm{m}^{2}\right)$, weight for age, height for age, and body mass index for age $z$ scores were calculated and recorded according to World Health Organization (WHO) standards using AnthroPlus software for personal computers) (WHO 2011).

2. Assessment of Tanner stage using Tanner classification (Tanner 1976).
3. Measurement of blood pressure $(\mathrm{mmHg})$ was done by a mercury sphygmomanometer.

B. Examinations done for patients for evidence of any diabetic complications:

1. Complete examination including chest, heart, abdomen, and full neurological examination for evidence of any diabetic complications.

2. Fundus examination by direct ophthalmoscope through dilated pupils for assessment of diabetic retinopathy.

III. Laboratory investigations: Routine investigations for all cases were done

1. Random blood glucose with the calculation of the mean value over the last year prior to the study.

2. HbA1c, the mean value over the last year was calculated.

3. Quantitative determination of urinary microalbumin for detection of diabetic nephropathy. Microalbuminuria was defined as the excretion rate (30-300 $\mathrm{mg} /$ urinary creatinine).

IV. Psychosocial assessment:

1. Assessment of family socioeconomic standard by the socioeconomic level of the family scale (Egyptian version) (El-Shakhs 2013)

2. Assessment of health-related quality of life (HRQOL) by the Pediatric Quality of Life Inventory $^{\mathrm{mm}}\left(\right.$ Peds $\mathrm{QL} \mathrm{L}^{\mathrm{mm}}$ ), one of the international tools for assessment of HRQOL of children (Varni et al. 1999). PedsQL ${ }^{\mathrm{m}}$ instrument provides both PedsQL ${ }^{\text {mo }}$ 4.0 Generic Core Scales (for healthy and diseased children) (Varni et al. 2001) and the PedsQL ${ }^{\mathrm{ma}}$ 3.0 Diabetes Module (for diabetic children) (Varni et al. 2003), having good reliability and validity and used together.

- PedsQL 4.0 Generic Core Scales (GCS) includes 23 items assessing 4 subscales (physical, emotional, social, and school functioning) with 8, 5, 5, and 5 items, respectively. Response scale with five categories, ranging from never a problem (0) to almost always a problem (Abuawad 2013). PedsQL ${ }^{\mathrm{m}}$ 3.0 diabetes module (DM) includes 28 questions assessing five subscales (diabetes symptoms, treatments barriers, treatment adherence, worry, and communications) with 11, 4, 7, 3, and 3 items, respectively. Its response scale is similar to that of the PedsQL ${ }^{\mathrm{m}} 4.0$ (GCS).

- Both child self-report and parent proxy-report of the two appropriate forms were used in the present study according to the child age (reports for children ( $8-12$ years) and adolescents (13-18 years). 


\section{Statistical analysis}

All data were coded, entered, and processed on an IBMPC compatible computer using SPSS (version 22). The quantitative data were presented as mean, standard deviations, and ranges as their distribution found parametric, while qualitative data were presented as number and percentages. The comparison between two independent groups with quantitative data and parametric distribution was done by using independent $t$ test, and data with non-parametric distribution was done by Mann-Whitney test. The comparison between more than two independent groups with quantitative data and parametric distribution was done by using one-way ANOVA and post hoc analyses using LSD tests. Correlation analysis: assessing the strength of association between two variables. The correlation coefficient denoted symbolically $r$, defined the strength and direction of the linear relationship between two variables. The confidence interval $(\mathrm{CI})$ was set to $95 \%$, and the margin of error accepted was set to $5 \%$. So, the $p$ value was considered significant as the following: $p>0.05=$ non-significant,$\quad p<0.05=$ significant and $p<0.01=$ highly significant

\section{Results}

The present study included 60 diabetic and 60 non-diabetic (age- and sex-matched) Egyptian children and adolescents with a mean age $(13.03 \pm 2.78$ and $12.43 \pm 2.66$ years), respectively. Table 1 shows some important descriptive demographic, clinical, and laboratory data of the studied diabetic and control groups. Thirty (five children and 25 adolescents) out of 60 patients had one or more microvascular complications; the most common chronic complication encountered in the studied patients was diabetic microalbuminuria being in 22 (13 patients had nephropathy alone and nine patients had nephropathy with other complications) representing $36.7 \%$ of all diabetic patients, followed by peripheral neuropathy being in 16 patients (eight patients had neuropathy alone and eight patients had neuropathy with other complications) representing $26.7 \%$ of all diabetic patients and retinopathy being in five patients (one patient had retinopathy with nephropathy and four patients had retinopathy with nephropathy and neuropathy) representing $8.3 \%$ of all diabetic patients (Fig. 1).

There was no significant difference between complicated and non-complicated patients regarding the number of DKA and hypoglycemic attacks $(p>0.05)$, despite of being higher in complicated patients (Table 2).

There was significant difference $(p<0.05)$ between complicated and non-complicated patients regarding age of onset of disease, disease duration, mean random blood glucose, HbA1c, and mean insulin dose, being higher in complicated patients except for age of onset of disease (that means the occurrence of diabetes was at younger age in diabetic patients with microvascular complications and they had poor metabolic control indices than that of non-complicated patients).

There was no significant difference between studied diabetic and healthy children (8-12 years) concerning their PedsQL ${ }^{\mathrm{mw}}$ 4.0 Generic Core Scales (GCS) (Table 3).

There was a significant decrease in PedsQL ${ }^{\mathrm{mm}} 4.0$ Generic Core Scales (GCS) for studied diabetic adolescents (13-18 years) compared to healthy adolescents.

There was no significant difference between diabetic male and female children (8-12 years) concerning their PedsQL ${ }^{\mathrm{Tm}}$ 4.0 Generic Core Scales (GCS) and PedsQL ${ }^{\mathrm{mm}}$ 3.0 Diabetic Module. There was a significant decrease in emotional functioning, social functioning, worry, and

Table 1 Some important descriptive demographic, clinical, and laboratory data of the studied diabetic and control groups

\begin{tabular}{|c|c|c|}
\hline Variables & $\begin{array}{l}\text { Diabetic group } \\
n=60 \\
\text { Mean } \pm S D \\
\text { Range }\end{array}$ & $\begin{array}{l}\text { Control group } \\
n=60 \\
\text { Mean } \pm \text { SD } \\
\text { Range }\end{array}$ \\
\hline Age (years) & $13.03 \pm 2.78(8-18)$ & $12.43 \pm 2.66(8-18)$ \\
\hline Age of onset of disease (years) & $5.68 \pm 2.77(1-12)$ & \\
\hline Disease duration (years) & $7.34 \pm 4.24(1-17)$ & \\
\hline Height (cm) & $151.23 \pm 10.17(125-167)$ & $150.00 \pm 12.51(120-172)$ \\
\hline Weight (kg) & $45.29 \pm 12.54(21-70)$ & $42.16 \pm 10.46(22-72)$ \\
\hline $\mathrm{BMI}\left(\mathrm{kg} / \mathrm{m}^{2}\right)$ & $19.33 \pm 3.31(13.4-27.3)$ & $18.35 \pm 2.51(12.1-28.1)$ \\
\hline Mean Insulin dose (IU/kg/d) & $1.04 \pm 0.30(0.48-1.70)$ & \\
\hline Systolic blood pressure (mmHg) & $110.33 \pm 10.73(90-130)$ & $104.58 \pm 10.05(90-120)$ \\
\hline Diastolic blood pressure $(\mathrm{mmHg})$ & $71.75 \pm 9.69(50-90)$ & $65.00 \pm 6.57(50-80)$ \\
\hline MRBG (mg/dl) & $214.15 \pm 49.94(105-360)$ & $84.53 \pm 9.25(70-105)$ \\
\hline $\mathrm{HbA}_{\mathrm{f}} \mathrm{c}(\%)$ & $8.88 \pm 1.56(5.6-12.6)$ & \\
\hline
\end{tabular}

$S D$ standard deviation, $I U / \mathrm{kg} / \mathrm{d}$ international unit per kilogram per day, $B M I$ body mass index, $M R B G$ mean random blood glucose, $M g / d l$ milligram per deciliter, $H b A_{1} c$ glycated hemoglobin $A_{1 C}$ 


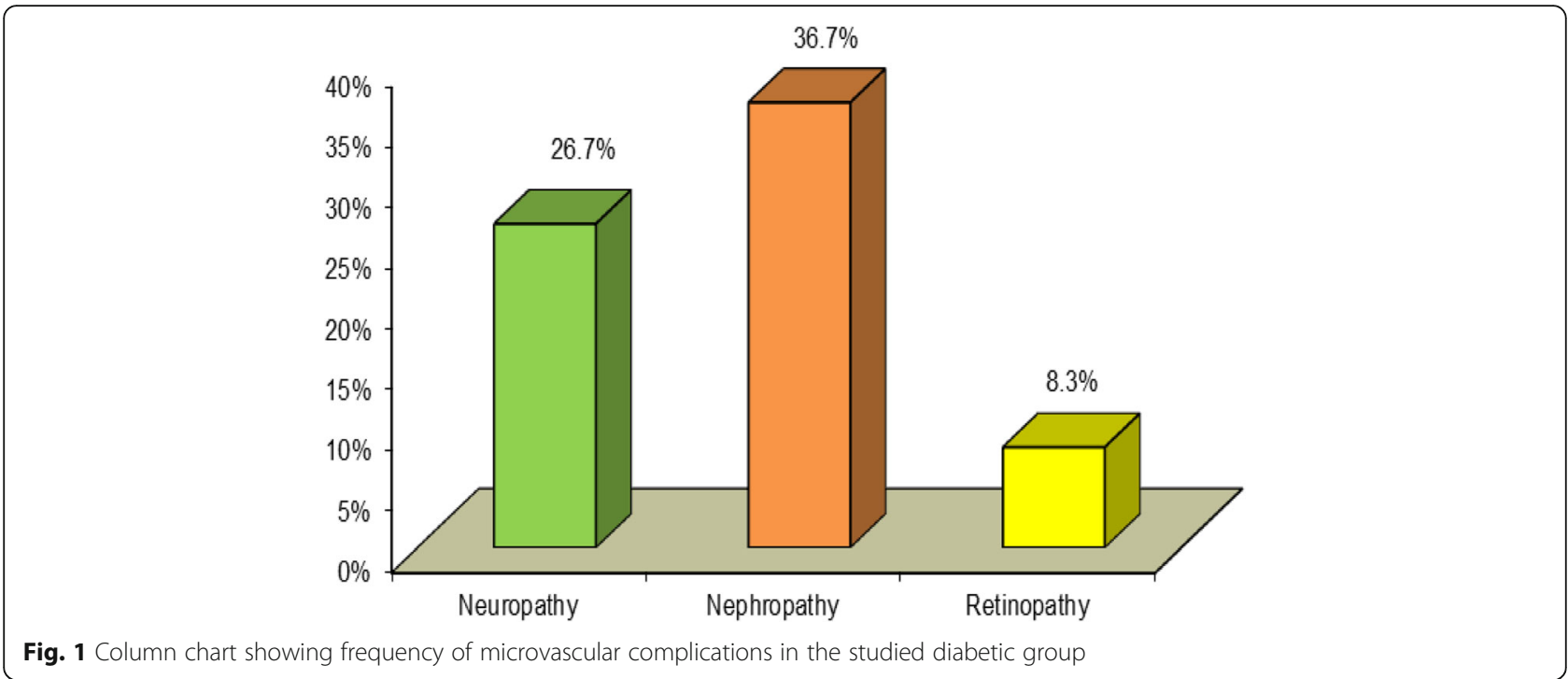

communication scales for studied diabetic female adolescents (13-18 years) compared to diabetic male adolescents (Table 4).

Table 5 and Fig. 2 indicate a significant decrease in all PedsQL ${ }^{\mathrm{Tm}}$ scales of the complicated group compared to the non-complicated group except for communication and diabetes symptom scales. There was a significant difference between non-complicated and control groups regarding their PedsQL ${ }^{\mathrm{Tm}}$ 4.0 GCS. Multiple regressions show that neuropathy was the most correlated complication with a total score of HRQOL $(p<0.0001)$.

Table 6 shows that Pediatric Quality of Life Inventory subscales and total scales scores were found to have negative statistically significant correlations with age, disease duration, BMI, SBP, and DBP in diabetic group and positive statistically significant correlations with age of onset of diabetes (for all PedsQL ${ }^{\mathrm{ma}} 4.0$ Generic Core Scale and only for the treatment adherence of the PedsQL ${ }^{\mathrm{ma}} 3.0$ Diabetic Module) and socioeconomic status (the most correlated factor was maternal education as proved by multiple regression analysis, $p=0.019$ ) (Table 6).

Table 7 shows that Pediatric Quality of Life Inventory subscales and total scale scores were found to have negative statistically significant correlations with all metabolic control parameters (MRBG, HbA1c, number of DKA attacks, hypoglycemic attacks, and hospitalizations) in the studied diabetic patients, except for mean insulin dose that showed negative statistically significant correlation only with the diabetes symptom scale (Table 7).

\section{Discussion}

The most frequent chronic complication encountered in the studied patients was diabetic microalbuminuria (nephropathy alone and with other complications) being in 22 out of 60 patients representing $36.7 \%$, followed by peripheral neuropathy being in 16 patients representing $26.7 \%$, then retinopathy being in five patients representing

Table 2 Comparison between the two studied diabetic (non-complicated and complicated) groups in terms of their metabolic control indices

\begin{tabular}{|c|c|c|c|c|c|}
\hline \multirow[t]{2}{*}{ Variables } & & \multirow[t]{2}{*}{ Non-complicated $n=30$} & \multirow[t]{2}{*}{ Complicated $n=30$} & \multicolumn{2}{|c|}{ Statistical test } \\
\hline & & & & $t / z^{*}$ & $p$ value \\
\hline A.O.D (yrs) & Mean \pm SD Range & $7.50 \pm 2.15(3.5-12)$ & $3.87 \pm 2.03(1-8)$ & 6.725 & $<0.001(\mathrm{HS})$ \\
\hline D.D (yrs) & Mean \pm SD Range & $3.75 \pm 1.96(1-7.5)$ & $10.93 \pm 2.45(9-17)$ & 12.545 & $<0.001(\mathrm{HS})$ \\
\hline MRBG (mg/dl) & Mean \pm SD Range & $199.20 \pm 41.78(105-290)$ & $229.10 \pm 53.54(119-360)$ & 2.412 & $0.019(S)$ \\
\hline $\mathrm{HbA} 1 \mathrm{c}(\%)$ & Mean \pm SD Range & $8.33 \pm 1.35(5.6-10.7)$ & $9.43 \pm 1.58(7-12.6)$ & 2.900 & $0.005(\mathrm{HS})$ \\
\hline Mean insulin dose $(\mathrm{IU} / \mathrm{kg} / \mathrm{d})$ & Mean \pm SD Range & $0.92 \pm 0.27(0.48-1.54)$ & $1.15 \pm 0.27(0.75-1.7)$ & 3.288 & $0.002(H S)$ \\
\hline DKA attacks (n/year) & Median (IQR) Range & $0(0-0)(0-2)$ & $0(0-1)(0-2)$ & $1.691^{*}$ & 0.091 (NS) \\
\hline Hypoglycemic attacks (n/year) & Median (IQR) Range & $0(0-1)(0-10)$ & $0.5(0-3)(0-5)$ & $1.718^{*}$ & $0.086(N S)$ \\
\hline
\end{tabular}

$t$ for independent $t$ test. $z$ for Mann-Whitney test

NS non-significant $(p>0.05), S$ significant $(p<0.05)$, HS highly significant $(p<0.01), I Q R$ interquartile range, A.O.D age of onset of disease, D.D disease duration, Yrs. years, MRBG mean random blood glucose, $\mathrm{mg} / \mathrm{dl}$ milligram per deciliter, IU/ $\mathrm{kg} / \mathrm{d}$ international unit per kilogram per day, $D K A$ diabetic ketoacidosis 
Table 3 Comparison between the studied diabetic children (8-12 years) and adolescents (13-18 years) with their corresponding control groups concerning their child self-reports and parent proxy-reports of PedsQL ${ }^{\mathrm{TM}} 4.0$ Generic Core Scales (GCS) scores

\begin{tabular}{|c|c|c|c|c|c|c|c|c|c|}
\hline \multirow[t]{2}{*}{ Functioning scale } & \multirow{2}{*}{$\begin{array}{l}\text { No. } \\
\text { of } \\
\text { items }\end{array}$} & \multirow{2}{*}{$\begin{array}{l}\text { Diabetic } \\
\text { children (8- } \\
12 \text { years) } \\
n=24\end{array}$} & \multirow{2}{*}{$\begin{array}{l}\text { Healthy } \\
\text { children (8- } \\
12 \text { years) } \\
n=28\end{array}$} & \multicolumn{2}{|c|}{ Independent $t$ test } & \multirow{2}{*}{$\begin{array}{l}\text { Diabetic } \\
\text { adolescents } \\
\text { (13-18 years) } \\
n=36\end{array}$} & \multirow{2}{*}{$\begin{array}{l}\text { Healthy } \\
\text { adolescents } \\
\text { (13-18 years) } \\
n=32\end{array}$} & \multicolumn{2}{|c|}{ Independent $t$ test } \\
\hline & & & & $t$ & $p$ value & & & $t$ & $p$ value \\
\hline \multicolumn{10}{|c|}{ PedsQL $L^{\text {тM }} 4.0$ Generic Core Scales (child self-report) } \\
\hline \multirow[t]{2}{*}{ Total score } & 23 & $77.05 \pm 14.58$ & $79.32 \pm 11.15$ & 0.638 & 0.527 & $64.37 \pm 14.54$ & $74.74 \pm 13.34$ & 3.051 & $0.003^{* *}$ \\
\hline & & $40.22-100$ & $55.43-100$ & & & $35.87-92.39$ & $45.66-92.39$ & & \\
\hline \multirow[t]{2}{*}{ Physical health } & 8 & $80.07 \pm 15.01$ & $82.6 \pm 11.01$ & 0.698 & 0.488 & $66.64 \pm 15.56$ & $80.20 \pm 13.63$ & 3.800 & $0.001^{* *}$ \\
\hline & & $40.63-100$ & $54.17-100$ & & & $37.5-96.88$ & $43.75-100$ & & \\
\hline \multirow[t]{2}{*}{ Emotional functioning } & 5 & $70.31 \pm 16.14$ & $74.67 \pm 19.41$ & 0.872 & 0.388 & $56.08 \pm 16.71$ & $65.07 \pm 19.48$ & 2.048 & $0.045^{*}$ \\
\hline & & $35-100$ & $35-100$ & & & $25-85$ & $33.33-100$ & & \\
\hline \multirow[t]{2}{*}{ Social functioning } & 5 & $81.35 \pm 13.89$ & $81.8 \pm 16.42$ & 0.105 & 0.917 & $68.06 \pm 15.70$ & $77.01 \pm 16.84$ & 2.267 & $0.027^{*}$ \\
\hline & & $45-100$ & $50-100$ & & & $35-90$ & $45-100$ & & \\
\hline \multirow[t]{2}{*}{ School functioning } & 5 & $74.48 \pm 15.78$ & $76.47 \pm 14.08$ & 0.482 & 0.632 & $65.52 \pm 13.67$ & $75.89 \pm 16.16$ & 2.864 & $0.006^{* *}$ \\
\hline & & $40-100$ & $50-100$ & & & $35-95$ & $40-100$ & & \\
\hline \multirow[t]{2}{*}{ Psychosocial health } & 15 & $75.54 \pm 14.89$ & $77.64 \pm 12.84$ & 0.547 & 0.587 & $63.27 \pm 14.48$ & $72.67 \pm 13.88$ & 2.725 & $0.008^{* *}$ \\
\hline & & $40-100$ & $45-100$ & & & $35-90$ & $44.23-94.23$ & & \\
\hline \multicolumn{10}{|c|}{ PedsQL ${ }^{\mathrm{TM}} 4.0$ Generic Core Scales (parent proxy-report) } \\
\hline \multirow[t]{2}{*}{ Total score } & 23 & $73.45 \pm 15.25$ & $77.75 \pm 10.82$ & 1.185 & 0.242 & $61.71 \pm 14.71$ & $71.97 \pm 11.96$ & 3.133 & $0.003^{* *}$ \\
\hline & & $39.13-98.91$ & $52.38-97.83$ & & & $32.61-86.96$ & $41.3-93.48$ & & \\
\hline \multirow[t]{2}{*}{ Physical health } & 8 & $76.63 \pm 16.14$ & $82.23 \pm 12.14$ & 1.427 & 0.160 & $64.94 \pm 16.76$ & $76.51 \pm 15.84$ & 2.915 & $0.005^{* *}$ \\
\hline & & $37.5-100$ & $50-100$ & & & $34.38-93.75$ & $37.5-96.88$ & & \\
\hline \multirow[t]{2}{*}{ Emotional functioning } & 5 & $67.92 \pm 17.87$ & $70.45 \pm 17.13$ & 0.519 & 0.606 & $52.19 \pm 16.73$ & $62.58 \pm 16.75$ & 2.555 & $0.013^{*}$ \\
\hline & & $30-100$ & $35-95$ & & & $20-80$ & $25-100$ & & \\
\hline \multirow[t]{2}{*}{ Social functioning } & 5 & $77.03 \pm 12.85$ & $82.5 \pm 12.95$ & 1.524 & 0.134 & $65.17 \pm 15.17$ & $74.71 \pm 15.29$ & 2.578 & $0.012^{*}$ \\
\hline & & $50-100$ & $60-100$ & & & $30-90$ & $40-100$ & & \\
\hline \multirow[t]{2}{*}{ School functioning } & 5 & $70.94 \pm 15.86$ & $73.57 \pm 14.33$ & 0.629 & 0.532 & $62.88 \pm 13.04$ & $72.25 \pm 14.43$ & 2.813 & $0.006^{* *}$ \\
\hline & & $35-100$ & $40-100$ & & & $30-90$ & $43.75-100$ & & \\
\hline \multirow[t]{2}{*}{ Psychosocial health } & 15 & $71.96 \pm 15.17$ & $75.4 \pm 11.35$ & 0.932 & 0.356 & $60.08 \pm 14.21$ & $69.98 \pm 12.39$ & 3.046 & $0.003^{* *}$ \\
\hline & & 40-98.33 & $50-98.33$ & & & $31.67-83.33$ & $36.67-91.67$ & & \\
\hline
\end{tabular}

*Significant $(p<0.05) .{ }^{*}$ Highly significant $(p<0.01)$

$8.3 \%$ of all diabetic patients. This came in accordance with what was reported by Gross et al. 2005, De Boer et al. 2011, and ADA 2018 that microalbuminuria was a common diagnosis in the clinical care of patients with type 1 diabetes mellitus, with a lifetime cumulative incidence of $20 \%$ to $40 \%$.

In the present study concerning the PedsQL ${ }^{\mathrm{m}} 4.0$ Generic Core Scales (GCS), there was no significant difference between studied diabetic and healthy children (8-12 years) (GCS total score mean $=77.05 \pm 14.58$ vs. $79.32 \pm 11.15$, respectively). But there was a significant decrease for studied diabetic adolescents (13-18 years) compared to healthy peers $(64.37 \pm 14.54$ vs. $74.74 \pm$ 13.34, respectively).

The decrease in general quality of life score in the group of diabetic adolescents might be due to the high percent of microvascular complications (25 out of 36 adolescents had one or more microvascular complications, representing $69.4 \%$ of the adolescent group) among them. Furthermore, the teen challenges faced with attempts of diabetes control. There were many studies that came in accordance with the current study findings, as for children, from which the study of Nieuwesteeg et al. (2012) who reviewed a systematic literature; 17 studies were eligible for these reviews, which have compared generic quality of life of children and adolescents with T1DM with that of healthy peers and revealed that, although children and adolescents with T1DM have to live with a demanding treatment regime, overall results revealed that their generic QOL scores were not impaired compared to healthy peers. However, disease-specific QOL problems, including a negative impact of diabetes 
Table 4 Differentiation between male and female diabetic children (8-12 years) and adolescents (13-18 years) concerning their PedsQL ${ }^{\text {TM }} 4.0$ Generic Core Scales (GCS) and PedsQL ${ }^{\text {TM }}$ 3.0 Diabetic Module

\begin{tabular}{|c|c|c|c|c|c|c|c|c|c|}
\hline \multirow[t]{2}{*}{ Functioning scale } & \multirow{2}{*}{$\begin{array}{l}\text { No. } \\
\text { of } \\
\text { items }\end{array}$} & \multirow[b]{2}{*}{$\begin{array}{l}\text { Diabetic } \\
\text { male } \\
\text { children (8- } \\
12 \text { years) } \\
n=12 \\
\text { Mean } \pm S D \\
\text { Range }\end{array}$} & \multirow[b]{2}{*}{$\begin{array}{l}\text { Diabetic } \\
\text { female } \\
\text { children (8- } \\
12 \text { years) } \\
n=12 \\
\text { Mean } \pm S D \\
\text { Range }\end{array}$} & \multicolumn{2}{|c|}{ Independent $t$ test } & \multirow[b]{2}{*}{$\begin{array}{l}\text { Diabetic } \\
\text { male } \\
\text { adolescents } \\
\text { (13- } \\
18 \text { years) } \\
n=10 \\
\text { Mean } \pm \text { SD } \\
\text { Range }\end{array}$} & \multirow[b]{2}{*}{$\begin{array}{l}\text { Diabetic } \\
\text { female } \\
\text { adolescents } \\
\text { (13- } \\
18 \text { years) } \\
n=26 \\
\text { Mean } \pm S D \\
\text { Range }\end{array}$} & \multicolumn{2}{|c|}{ Independent $t$ test } \\
\hline & & & & $t$ & $p$ value & & & $t$ & $p$ value \\
\hline \multicolumn{10}{|c|}{ PedsQL ${ }^{\mathrm{TM}} 4.0$ Generic Core Scales } \\
\hline \multirow[t]{2}{*}{ Total score } & 23 & $79.55 \pm 14.50$ & $74.55 \pm 14.85$ & 0.834 & 0.413 & $70.56 \pm 11.47$ & $61.99 \pm 15.09$ & 1.619 & 0.115 \\
\hline & & $53.41-100$ & $40.22-94.57$ & & & $(50-84.09)$ & $(35.87-92.39)$ & & \\
\hline \multirow[t]{2}{*}{ Physical health } & 8 & $82.33 \pm 13.08$ & $77.81 \pm 17.00$ & 0.730 & 0.474 & $70.22 \pm 13.07$ & $65.27 \pm 16.44$ & 0.852 & 0.400 \\
\hline & & $56.25-100$ & $40.63-100$ & & & $(45.83-84.38)$ & $(37.5-96.88)$ & & \\
\hline \multirow[t]{2}{*}{ Emotional functioning } & 5 & $73.85 \pm 17.08$ & $66.77 \pm 15.02$ & 1.079 & 0.293 & $65.38 \pm 13.39$ & $52.50 \pm 16.69$ & 2.406 & $0.026^{*}$ \\
\hline & & $45-100$ & $35-90$ & & & $(40-80)$ & $(25-85)$ & & \\
\hline \multirow[t]{2}{*}{ Social functioning } & 5 & $83.75 \pm 14.32$ & $78.96 \pm 13.63$ & 0.840 & 0.410 & $79.75 \pm 8.54$ & $63.56 \pm 15.6$ & 3.092 & $0.004^{* *}$ \\
\hline & & $50-100$ & $45-95$ & & & $(65-90)$ & $(35-90)$ & & \\
\hline \multirow[t]{2}{*}{ School functioning } & 5 & $77.08 \pm 17.12$ & $71.88 \pm 14.58$ & 0.802 & 0.431 & $66.13 \pm 13.00$ & $65.29 \pm 14.17$ & 0.162 & 0.872 \\
\hline & & $45-100$ & $40-100$ & & & $(43.75-85)$ & $(35-95)$ & & \\
\hline \multirow[t]{2}{*}{ Psychosocial health } & 15 & $78.26 \pm 15.70$ & $72.82 \pm 14.17$ & 0.892 & 0.382 & $70.68 \pm 10.98$ & $60.41 \pm 14.82$ & 1.983 & 0.055 \\
\hline & & $46.67-100$ & $40-91.67$ & & & $(51.67-83.93)$ & $(35-90)$ & & \\
\hline \multicolumn{10}{|c|}{ PedsQL ${ }^{\text {TM }} 3.0$ Diabetic Module } \\
\hline \multirow[t]{2}{*}{ total score } & 28 & $76.12 \pm 15.39$ & $69.25 \pm 15.89$ & 1.076 & 0.294 & $65.60 \pm 12.29$ & $58.69 \pm 15.93$ & 1.233 & 0.226 \\
\hline & & $50-97.32$ & $37.04-88.39$ & & & $(41.35-75.93)$ & $(29.63-87.96)$ & & \\
\hline \multirow[t]{2}{*}{ Diabetes symptoms } & 11 & $72.86 \pm 16.66$ & $67.52 \pm 17.12$ & 0.775 & 0.447 & $63.18 \pm 16.06$ & $57.91 \pm 17.00$ & 0.846 & 0.403 \\
\hline & & $45.45-93.18$ & $31.82-84.1$ & & & $(31.82-79.55)$ & $(25-86.36)$ & & \\
\hline \multirow[t]{2}{*}{ Treatment barriers } & 4 & $70.31 \pm 19.61$ & $63.19 \pm 16.05$ & 0.973 & 0.341 & $61.04 \pm 10.72$ & $55.69 \pm 16.28$ & 0.958 & 0.345 \\
\hline & & $43.75-100$ & $37.5-87.5$ & & & $(41.66-68.75)$ & $(31.25-87.5)$ & & \\
\hline \multirow[t]{2}{*}{ Treatment adherence } & 7 & $82.54 \pm 13.41$ & $76.19 \pm 15.25$ & 1.083 & 0.290 & $67.86 \pm 11.17$ & $65.93 \pm 14.44$ & 0.379 & 0.707 \\
\hline & & $57.14-100$ & $46.43-100$ & & & $(42.86-78.57)$ & (32.14-89.29) & & \\
\hline \multirow[t]{2}{*}{ Worry } & 3 & $76.39 \pm 15.52$ & $64.93 \pm 19.01$ & 1.617 & 0.120 & $69.58 \pm 10.02$ & $50.64 \pm 19.25$ & 2.944 & $0.006^{* *}$ \\
\hline & & $41.67-100$ & $25-91.67$ & & & $(50-83.33)$ & $(16.67-87.5)$ & & \\
\hline \multirow[t]{2}{*}{ Communication } & 3 & $81.60 \pm 13.35$ & $70.83 \pm 13.99$ & 1.928 & 0.067 & $71.67 \pm 10.72$ & $56.09 \pm 18.11$ & 3.172 & $0.004^{* *}$ \\
\hline & & $58.33-100$ & $41.67-83.33$ & & & $(50-87.5)$ & $(16.67-91.67)$ & & \\
\hline
\end{tabular}

on daily functioning and diabetes-related worries, were certainly present. Recently, Murillo et al. (2017b) showed that children and adolescents with T1DM reported similar HRQOL as the general population of the same age and gender, although slightly worse physical well-being than their healthy peers. Also, the study of AlBuhairan et al. 2016 was in accordance with the present study findings, as for adolescents, they reported a mean score of (64.8) indicating poor general HRQOL after conducting a cross-sectional study of the quality of life of 315 diabetic adolescents (12-18 years) in Saudi Arabia.

In contrast, some researchers reported worse quality of life for people with diabetes compared to the general population, especially regarding physical functioning and well-being, such as the case-control study done by (Abdul-Rasoul et al. 2013) on 436 diabetic children and adolescents (2-18 years) in Kuwait, who filled the PedsQL ${ }^{\mathrm{Tm}}$ 4.0 Generic Core Scales (GCS) and PedsQL 3.0 Diabetes Module. Their study revealed that children with T1DM reported lower total generic QOL $(75.6 \pm 8.9)$ than controls $(83.5 \pm$ 9.5, $p=0.001)$. The QOL scores were lower for physical $(75.1 \pm 11.6$ vs. $84.2 \pm 9.3, p<0.05)$ and emotional $(72.7 \pm 10.4$ vs. $84.1 \pm 9.1, p<0.001)$ domains. There was no difference in the school $(73.5 \pm 9.3$ vs. $73.7 \pm$ $8.1, p=0.15)$ and social $(83.2 \pm 8.8$ vs. $83.7 \pm 8.4, p=$ $0.13)$ QOL scores between patients and controls. And de Souza et al. 2015 who conducted a retrospective, 
Table 5 Comparison between complicated and non-complicated groups concerning their PedsQL ${ }^{\mathrm{TM}} 4.0$ Generic Core Scales (GCS) and PedsQL ${ }^{\text {TM }} 3.0$ Diabetic Module Scores

\begin{tabular}{|c|c|c|c|c|c|c|}
\hline \multirow{2}{*}{\multicolumn{2}{|c|}{ Functioning scale }} & \multirow[t]{2}{*}{ No. of items } & \multirow{2}{*}{$\begin{array}{l}\text { Non-complicated } \\
n=30\end{array}$} & \multirow{2}{*}{$\begin{array}{l}\text { Complicated } \\
n=30\end{array}$} & \multicolumn{2}{|c|}{ Independent $t$ test } \\
\hline & & & & & $t$ & $p$ value \\
\hline \multicolumn{7}{|c|}{ PedsQL ${ }^{\mathrm{TM}} 4.0$ Generic Core Scales } \\
\hline \multirow{2}{*}{\multicolumn{2}{|c|}{ Total score }} & 23 & $75.48 \pm 12.72$ & $63.40 \pm 16.32$ & 3.196 & $0.002^{* *}$ \\
\hline & & & $(40.22-100)$ & $(35.87-96.74)$ & & \\
\hline \multirow{2}{*}{\multicolumn{2}{|c|}{ Physical health }} & 8 & $79.12 \pm 12.62$ & $64.91 \pm 17.22$ & 3.644 & $0.001^{* *}$ \\
\hline & & & $(40.63-100)$ & $(37.5-96.88)$ & & \\
\hline \multirow{2}{*}{\multicolumn{2}{|c|}{ Emotional functioning }} & 5 & $68.50 \pm 14.92$ & $55.04 \pm 18.11$ & 3.141 & $0.003^{* *}$ \\
\hline & & & $(35-100)$ & $(25-95)$ & & \\
\hline \multirow{2}{*}{\multicolumn{2}{|c|}{ Social functioning }} & 5 & $78.63 \pm 13.62$ & $68.13 \pm 17.20$ & 2.621 & $0.011^{*}$ \\
\hline & & & $(45-100)$ & $(35-100)$ & & \\
\hline \multirow{2}{*}{\multicolumn{2}{|c|}{ School functioning }} & 5 & $73.92 \pm 13.19$ & $64.29 \pm 15.52$ & 2.588 & $0.012^{*}$ \\
\hline & & & $(40-100)$ & $(35-100)$ & & \\
\hline \multirow{2}{*}{\multicolumn{2}{|c|}{ Psychosocial health }} & 15 & $73.72 \pm 13.25$ & $62.63 \pm 16.26$ & 2.897 & $0.005^{* *}$ \\
\hline & & & $(40-100)$ & $(35-98.33)$ & & \\
\hline \multicolumn{7}{|c|}{ PedsQL ${ }^{\text {TM }} 3.0$ Diabetic Module } \\
\hline \multirow{2}{*}{\multicolumn{2}{|c|}{ Total score }} & 28 & $70.13 \pm 14.44$ & $60.75 \pm 17.07$ & 2.296 & $0.025^{*}$ \\
\hline & & & $(37.04-97.32)$ & $(29.63-93.75)$ & & \\
\hline \multirow{2}{*}{\multicolumn{2}{|c|}{ Diabetes symptoms }} & 11 & $68.01 \pm 15.14$ & $59.39 \pm 18.69$ & 1.963 & 0.055 \\
\hline & & & (31.82-93.18) & $(25-90.91)$ & & \\
\hline \multirow{2}{*}{\multicolumn{2}{|c|}{ Treatment barriers }} & 4 & $65.42 \pm 16.97$ & $56.60 \pm 15.58$ & 2.097 & $0.040^{*}$ \\
\hline & & & $(31.25-100)$ & $(31.25-87.5)$ & & \\
\hline \multirow{2}{*}{\multicolumn{2}{|c|}{ Treatment adherence }} & 7 & $77.42 \pm 12.61$ & $65.83 \pm 15.43$ & 3.184 & $0.002^{* *}$ \\
\hline & & & $(46.43-100)$ & $(32.14-100)$ & & \\
\hline \multirow{2}{*}{\multicolumn{2}{|c|}{ Worry }} & 3 & $66.94 \pm 16.98$ & $56.67 \pm 21.46$ & 2.057 & $0.044^{*}$ \\
\hline & & & $(25-100)$ & $(16.67-91.67)$ & & \\
\hline \multirow{2}{*}{\multicolumn{2}{|c|}{ Communication }} & 3 & $70.14 \pm 16.85$ & $63.33 \pm 19.00$ & 1.468 & 0.147 \\
\hline & & & $(25-100)$ & $(16.67-100)$ & & \\
\hline \multirow[t]{2}{*}{ Parameters } & Non-complicated & Complicated & Control group & \multicolumn{3}{|c|}{ One-way ANOVA } \\
\hline & & & & F & $p$ value & \\
\hline GCS total score & $75.48 \pm 12.7240 .22-100$ & $63.40 \pm 16.32$ 35.87-96.74 & $76.88 \pm 12.4845 .66-100$ & 10.417 & $<0.001^{* *}$ & \\
\hline \multicolumn{7}{|c|}{ Post hoc analysis using LSD test } \\
\hline Comparisons & Non-complicated vs. complicated & \multicolumn{2}{|l|}{ Non-complicated vs. control } & \multicolumn{3}{|c|}{ Complicated vs. control } \\
\hline$p$ value & $<0.001^{* *}$ & 0.645 & & $<0.001^{* *}$ & & \\
\hline
\end{tabular}

cross-sectional, and multicenter study performed by the Brazilian Type 1 Diabetes Study Group that analyzed Euro QOL scores from 3005 participants with type $1 \mathrm{DM}$ in 28 public clinics in Brazil.

In the present study, there were not significant differences between diabetic male and female children (8-12 years) concerning their PedsQL ${ }^{\mathrm{Tm}} 4.0$ Generic Core Scales (GCS) and PedsQL ${ }^{\mathrm{m}}$ 3.0 Diabetic Module Scores. There was a significant decrease in the emotional functioning, social functioning, worry, and communication scales for studied diabetic female adolescents (13-18 years) compared to diabetic male adolescents; all scores of females were lower than males.

A number of researchers have reported that quality of life is better among diabetic boys than diabetic girls, and boys are more satisfied with their diabetes treatment regimen, having lower diabetes burden (Forsander et al. 2017). The same finding reported by recent studies of Murillo et al. 2017b and Anderson et al. 2017 that enrolled lower HRQOL scores in virtually all dimensions in diabetic girls. Another study was done in Egypt by M. Hassan et al. 2017 on 150 


\section{PedsQL ${ }^{\mathrm{TM}}$ 4.0 Generic Core Scales}

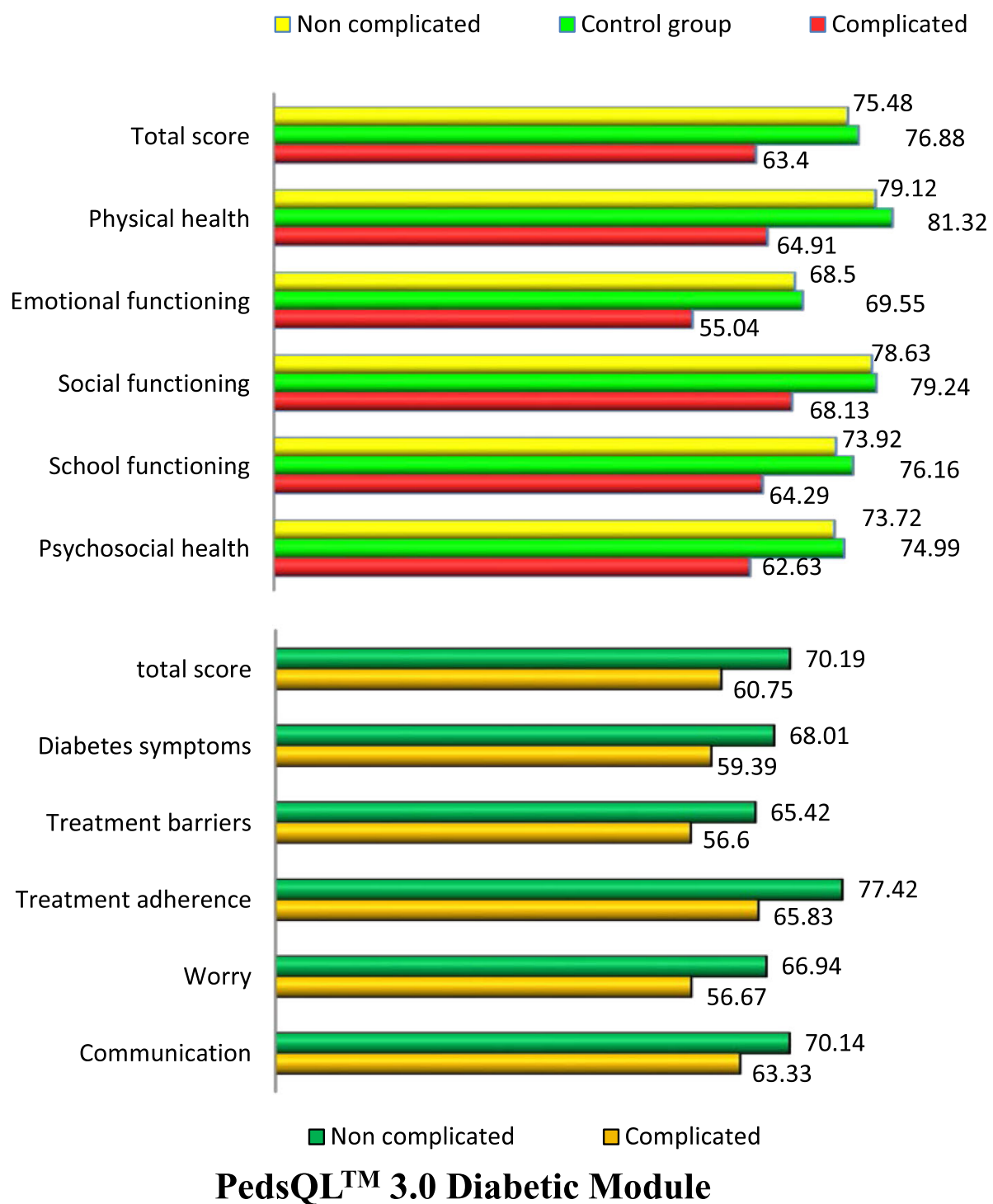

Fig. 2 Column chart showing comparison between complicated and non-complicated groups concerning their PedsQL $L^{\mathrm{TM}} 4.0$ Generic Core Scales and PedsQL ${ }^{\mathrm{TM}}$ 3.0 Diabetic Module Scores

adolescents (82 males and 68 females) (10-18 years), completing "Quality of Life for Youth" questionnaire at the Diabetes, Endocrine and Metabolism Pediatric Unit (DEMPU) clinic; males showed a significantly better mean QOL score than females $(p=0.004)$.

It is supposed that the decreased HRQOL in diabetic girls return to their general low perception of their QOL. Bisegger et al. 2005 reported gender differences as potential causes of greater vulnerability of teen girls for decreased HRQOL aspects than boys, from the age of 13 years on, besides being diabetic, such as the more drastic puberty for girls; the onset of menstruation, hormonal fluctuations (Toffol et al. 2014; Tanna et al. 2016), higher levels of perceived stress, and stress-related health complaints in adolescent girls than boys (Östberg et al. 2015), with handling problems and difficulties in different ways, namely girls tend to direct their coping patterns inwards, and boys outwards (Wiklund et al. 2012); and less body image satisfaction (Griffiths et al. 2017) and self-esteem (Gouveia et al. 2014), more sensitivity, empathic concern (Van der Graaff et al. 2014), and argument due to the social demands placed on girls, that are more staggering and more difficult to achieve (Barcellos et al. 2014). The difference in relationships with peers and social relations 
Table 6 Correlation between Pediatric Quality of Life Inventory scores with demographic and clinical characteristics in the studied diabetic patients

\begin{tabular}{|c|c|c|c|c|c|c|c|c|c|c|c|c|c|c|}
\hline \multirow[t]{2}{*}{ Functioning scale } & \multicolumn{2}{|c|}{ Age (years) } & \multicolumn{2}{|c|}{ A.O.D (years) } & \multicolumn{2}{|c|}{ D.D (years) } & \multicolumn{2}{|c|}{$\begin{array}{l}\text { Socioeconomic } \\
\text { score }\end{array}$} & \multicolumn{2}{|c|}{ BMI $\left(\mathrm{kg} / \mathrm{m}^{2}\right)$} & \multicolumn{2}{|c|}{$\begin{array}{l}\text { Systolic blood } \\
\text { pressure } \\
(\mathrm{mmHg})\end{array}$} & \multicolumn{2}{|c|}{$\begin{array}{l}\text { Diastolic blood } \\
\text { pressure }(\mathrm{mmHg})\end{array}$} \\
\hline & $r$ & $p$ value & $r$ & $\begin{array}{l}p \\
\text { value }\end{array}$ & r & $p$ value & $r$ & $p$ value & $r$ & $p$ value & $r$ & $\begin{array}{l}p \\
\text { value }\end{array}$ & $r$ & $p$ value \\
\hline \multicolumn{15}{|c|}{ 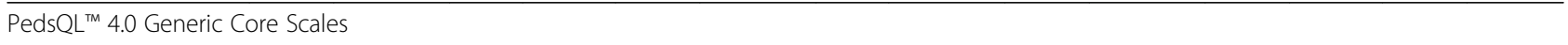 } \\
\hline Total score & $\overline{0.647}$ & $<0.001^{* *}$ & 0.311 & $0.016^{*}$ & $\overline{0} .630$ & $<0.001^{* *}$ & 0.551 & $<0.001^{* *}$ & $\begin{array}{l}- \\
0.577\end{array}$ & $<0.001^{* *}$ & $\overline{0} .379$ & $0.003^{* *}$ & $\overline{-} .475$ & $<0.001^{* *}$ \\
\hline Physical health & $\overline{0.646}$ & $<0.001^{* *}$ & 0.332 & $0.010^{* *}$ & $\begin{array}{l}- \\
0.643\end{array}$ & $<0.001^{* *}$ & 0.471 & $<0.001^{* *}$ & $\overline{0}-549$ & $<0.001^{* *}$ & $\begin{array}{l}- \\
0.382\end{array}$ & $0.003^{* *}$ & $\overline{0} .481$ & $<0.001^{* *}$ \\
\hline $\begin{array}{l}\text { Psychosocial } \\
\text { health }\end{array}$ & - & $<0.001^{* *}$ & 0.294 & $0.023^{*}$ & $-\overline{0.607}$ & $<0.001^{* *}$ & 0.583 & $<0.001^{* *}$ & $\begin{array}{l}- \\
0.577\end{array}$ & $<0.001^{* *}$ & $-\overline{0.368}$ & $0.004^{* *}$ & $\overline{0} .462$ & $<0.001^{* *}$ \\
\hline \multicolumn{15}{|c|}{ PedsQL ${ }^{\text {TM }} 3.0$ Diabetic Module } \\
\hline total score & $-\overline{0.608}$ & $<0.001^{* *}$ & 0.236 & 0.069 & - & $<0.001^{* *}$ & 0.545 & $<0.001^{* *}$ & $\begin{array}{l}- \\
0.498\end{array}$ & $<0.001^{* *}$ & $-\overline{0.296}$ & $0.021^{*}$ & $\begin{array}{l}- \\
0.407\end{array}$ & $0.001^{* *}$ \\
\hline $\begin{array}{l}\text { Diabetes } \\
\text { symptoms }\end{array}$ & $\begin{array}{l}- \\
0.560\end{array}$ & $<0.001^{* *}$ & 0.226 & 0.083 & $\begin{array}{l}- \\
0.515\end{array}$ & $<0.001^{* *}$ & 0.541 & $<0.001^{* *}$ & $\begin{array}{l}- \\
0.452\end{array}$ & $<0.001^{* *}$ & $\begin{array}{l}- \\
0.265\end{array}$ & $0.041^{*}$ & $\begin{array}{l}- \\
0.367\end{array}$ & $0.004^{* *}$ \\
\hline Treatment barriers & $-\overline{0.554}$ & $<0.001^{* *}$ & 0.229 & 0.078 & $-\overline{0.516}$ & $<0.001^{* *}$ & 0.541 & $<0.001^{* *}$ & $\overline{-} .422$ & $0.001^{* *}$ & $-\overline{0.226}$ & 0.082 & $-\overline{0.332}$ & $0.010^{* *}$ \\
\hline $\begin{array}{l}\text { Treatment } \\
\text { adherence }\end{array}$ & $-\overline{0.630}$ & $<0.001^{* *}$ & 0.305 & $0.018^{*}$ & $\begin{array}{l}- \\
0.614\end{array}$ & $<0.001^{* *}$ & 0.505 & $<0.001^{* *}$ & $\begin{array}{l}- \\
0.527\end{array}$ & $<0.001^{* *}$ & $\overline{-}-330$ & $0.010^{* *}$ & $-\overline{0.435}$ & $0.001^{* *}$ \\
\hline Worry & $\begin{array}{l}- \\
0.597\end{array}$ & $<0.001^{* *}$ & 0.167 & 0.201 & $\begin{array}{l}- \\
0.504\end{array}$ & $<0.001^{* *}$ & 0.488 & $<0.001^{* *}$ & $\begin{array}{l}- \\
0.522\end{array}$ & $<0.001^{* *}$ & $\overline{-}-314$ & $0.015^{*}$ & $\overline{0.439}$ & $<0.001^{* *}$ \\
\hline Communication & $\begin{array}{l}- \\
0.598\end{array}$ & $<0.001^{* *}$ & 0.096 & 0.465 & $\begin{array}{l}- \\
0.459\end{array}$ & $<0.001^{* *}$ & 0.496 & $<0.001^{* *}$ & $\begin{array}{l}- \\
0.509\end{array}$ & $<0.001^{* *}$ & $\begin{array}{l}- \\
0.310\end{array}$ & $0.016^{*}$ & $\begin{array}{l}- \\
0.397\end{array}$ & $0.002^{* *}$ \\
\hline
\end{tabular}

A.O.D age of onset of disease, D.D disease duration

${ }^{* *}$ Correlation is significant at the 0.01 level. * Correlation is significant at the 0.05 level

Table 7 Correlation between Pediatric Quality of Life Inventory scores with metabolic control parameters of type 1 diabetes in the studied diabetic patients

\begin{tabular}{|c|c|c|c|c|c|c|c|c|c|c|c|c|}
\hline \multirow[t]{2}{*}{ Functioning scale } & \multicolumn{2}{|c|}{ MRBG (mg/dl) } & \multicolumn{2}{|c|}{$\mathrm{HbA1c}(\%)$} & \multicolumn{2}{|c|}{$\begin{array}{l}\text { Mean insulin dose } \\
(\mathrm{IU} / \mathrm{kg} / \mathrm{d})\end{array}$} & \multicolumn{2}{|c|}{$\begin{array}{l}\text { Number of DKA } \\
\text { attacks }\end{array}$} & \multicolumn{2}{|c|}{$\begin{array}{l}\text { Number of } \\
\text { hypoglycemic } \\
\text { attacks }\end{array}$} & \multicolumn{2}{|c|}{$\begin{array}{l}\text { Number of } \\
\text { hospitalization }\end{array}$} \\
\hline & $r$ & $p$ value & $r$ & $p$ value & $r$ & $p$ value & $r$ & $p$ value & $r$ & $p$ value & $r$ & $p$ value \\
\hline \multicolumn{13}{|c|}{ PedsQL ${ }^{\mathrm{TM}} 4.0$ Generic Core Scales } \\
\hline Total score & -0.875 & $<0.001^{* *}$ & -0.890 & $<0.001^{* *}$ & -0.217 & 0.095 & -0.751 & $<0.001^{* *}$ & -0.580 & $<0.001^{* *}$ & -0.751 & $<0.001^{* *}$ \\
\hline Physical health & -0.881 & $<0.001^{* *}$ & -0.904 & $<0.001^{* *}$ & -0.179 & 0.171 & -0.758 & $<0.001^{* *}$ & -0.604 & $<0.001^{* *}$ & -0.758 & $<0.001^{* *}$ \\
\hline Psychosocial health & -0.847 & $<0.001^{* *}$ & -0.856 & $<0.001^{* *}$ & -0.227 & 0.082 & -0.726 & $<0.001^{* *}$ & -0.550 & $<0.001^{* *}$ & -0.726 & $<0.001^{* *}$ \\
\hline \multicolumn{13}{|c|}{ PedsQL ${ }^{\text {TM }}$ 3.0 Diabetic Module } \\
\hline total score & -0.925 & $<0.001^{* *}$ & -0.941 & $<0.001^{* *}$ & -0.247 & 0.057 & -0.750 & $<0.001^{* *}$ & -0.616 & $<0.001^{* *}$ & -0.750 & $<0.001^{* *}$ \\
\hline Diabetes symptoms & -0.916 & $<0.001^{* *}$ & -0.934 & $<0.001^{* *}$ & -0.285 & $0.027^{*}$ & -0.743 & $<0.001^{* *}$ & -0.645 & $<0.001^{* *}$ & -0.743 & $<0.001^{*}$ \\
\hline Treatment barriers & -0.869 & $<0.001^{* *}$ & -0.907 & $<0.001^{* *}$ & -0.236 & 0.070 & -0.633 & $<0.001^{* *}$ & -0.547 & $<0.001^{* *}$ & -0.633 & $<0.001^{* *}$ \\
\hline Treatment adherence & -0.940 & $<0.001^{* *}$ & -0.941 & $<0.001^{* *}$ & -0.240 & 0.065 & -0.735 & $<0.001^{* *}$ & -0.579 & $<0.001^{* *}$ & -0.735 & $<0.001$ \\
\hline Worry & -0.816 & $<0.001^{* *}$ & -0.826 & $<0.001^{* *}$ & -0.184 & 0.159 & -0.740 & $<0.001^{* *}$ & -0.561 & $<0.001^{* *}$ & -0.740 & $<0.001^{* *}$ \\
\hline Communication & -0.720 & $<0.001^{* *}$ & -0.738 & $<0.001^{* *}$ & -0.243 & 0.061 & -0.662 & $<0.001^{* *}$ & -0.479 & $<0.001^{* *}$ & -0.662 & $<0.001^{* *}$ \\
\hline
\end{tabular}


between girls and boys at the teenage (Rose and Asher 2017) is also that boys often have more privileges and are given more space than girls (Chawla 2004). Besides that, diabetic females trend to have worse metabolic control, higher frequency of microvascular complications, higher incidence of diabetic ketoacidosis (DKA), dyslipidemia, and weight problems than boys at adolescence (Forsander et al. 2017).

On the contrast, some studies reported no difference between diabetic girls and boys regarding their scores of HRQOL, as the studies done by Vanelli et al. 2003, AlAkour et al. 2010, Baş et al. 2011, and Arabiat and Al Jabery 2013. While, the recent Egyptian study done in Assiut city by Gadallah et al. 2017 reported that gender had no significant effect on any of the QOL aspects except for the school functions where girls reported significantly higher scores, explaining these findings due to Egyptian community, especially upper Egypt that do not give enough degree of freedom for females so they spend most of their times in studying, in addition to the preoccupation of males with other social activities.

The current study declared the adverse effects of diabetic microvascular complications on the HRQOL and well-being of type 1 diabetic children and adolescents, and the the most worsen effect was associated with neuropathy $(p<0.0001)$. Many other studies were in accord with these findings such as the study of Abolfotouh et al. 2011, Hannula et al. 2015, and Alvarado-Martel et al. 2015 who reported lower QOL scores associated with diabetic complications. Moreover, Jacobson et al. 2013 revealed that long-term chronic diabetic complications and their associated symptoms led to decreased HRQOL, with most worsen effect associated with neuropathy $(p<0.0001)$. The same finding was illustrated by Bai et al. 2017 that compared to other complications, neuropathy had the greatest association with distress and depression in longstanding T1DM, that in accordance with what was previously reported by Dobrota et al. 2014 that the quality of life was expectedly lower in diabetic patients suffering painful polyneuropathy, basically caused by painful symptoms, sleeping disorders, higher intensity of depressive symptoms in these dissatisfied patients and that all these conditions require additional efforts in terms of time and energy needed for treatment and lay a more drastically burden on subject's quality of life than the disease itself. On the other hand, Tofeeq et al. 2017 found an impact of both nephropathy and retinopathy on the physical domain of quality of life while there was no significant association between neuropathy and physical problems of children and adolescents with T1D.

In the current study, negative statistically significant correlations were found between HRQOL domains with demographic and clinical characteristics (age, disease duration, BMI, SBP, and DBP), and positive statistically significant correlations with socioeconomic status (especially maternal education, $p=0.019$ ) and age of onset of diabetes (for all PedsQL ${ }^{\mathrm{Tm}} 4.0$ Generic Core Scale and only for the treatment adherence of the PedsQL ${ }^{\text {тu }} 3.0$ Diabetic module) were illuminated in the studied diabetic patients. As regards the effect of disease duration on HRQOL, current results were in accord with the studies of Jafari et al. 2011, Hilliard et al. 2013, Abdul-Rasoul et al. 2013, and da Costa and Vieira 2015. The negative impact of long diabetes duration on quality of life might result from significant deterioration in glycemic control, deterioration in treatment adherence over this time, and occurrence of long-term diabetes complications that reduce person's perception of well-being and productivity. On the other hand, the studies of Stahl et al. 2012, Lawrence et al. 2012, Abd-Elall et al. 2016, and M. Hassan et al. 2017 stated that duration of diabetes had no statistically significant effect on QOL.

As regards the effect of socioeconomic status on HRQOL, current results were in accord with the study done by da Costa and Vieira 2015, reported an association between the lower education levels of parents and the deterioration of the QOL of diabetic children, as the lower education levels of parents may be associated with the insecurity of adolescents due to lack of information and the anticipation of socioeconomic difficulties in the future. Gesuita et al. 2017 found that a lower socioeconomic level was associated with poor metabolic control and QOL of young type 1 diabetic patients less than 18 years of age.

Regarding the effect of body mass index on HRQOL, current results were in agreement with the studies of Schwimmer et al. 2003 and Williams et al. 2005, but in contrast to the current findings, no significant differences in HRQOL by BMI category demonstrated by the studies of Naughton et al. 2008, Lawrence et al. 2012, and Hassan et al. 2017.

In the current study, negative statistically significant correlations were found between HRQOL domains (Pediatric Quality of Life Inventory scores) with all metabolic control parameters (MRBG, HbA1c, number of DKA, number of hypoglycemic attacks, and number of hospitalization due to acute diabetic complications); the strongest relation was between treatment adherence scale with HbA1c\% $\left(r=-0.941, p^{<} 0.001\right)$, but no significant correlation found with mean insulin dose in the studied diabetic patients.

As regards the effect of metabolic control (indicated mainly by $\mathrm{HbA} 1 \mathrm{c} \%$ ) on $\mathrm{HRQOL}$, current results were in agreement with many previous studies as those done by Hassan et al. 2006, Nansel et al. $2008(r=-0.08)$, Ingerski et al. $2010(r=-0.28)$, and Lawrence et al. 2012 $(r=-0.232, p<0.0001)$; all of them reported an inverse 
association between PedsQL-T1DM total score and HbA1c. In addition, Murillo et al. 2017a showed evidence that better HRQOL was associated with better metabolic control, and they found an association between HRQOL, HbA1c, and treatment adherence, explaining that adherence to treatment was very important to achieve a good metabolic control and was associated with better HRQOL, recommending that treatment adherence should be taken into account to improve HRQOL in diabetic patients and giving better outcome.

On the other hand, some studies did not come in context with the previous findings, such as the study conducted by Ingerski et al. 2010. On the other hand, Paula et al. 2017 found that mean glucose level (MGL), but not HbA1c or number of hypoglycemic episodes, was the glycemic control parameter that best correlated with short-term perception of HRQOL; in a sample of T1D patients with low socioeconomic status, as in this population, the perception of good daily glucose control may be more easily interpreted than biochemical parameters of glycemic control (HbA1c).

Regarding the correlation between the number of attacks of diabetic ketoacidosis and HRQOL, current results were in agreement with the previous study done by Frøisland et al. 2013 who reported that DKA significantly reduced the HRQOL of young T1DM patients. Also, Al-Hayek et al. 2014 in Saudi Arabia found that adolescents with DKA had significantly lower HRQOL than without DKA in all domains of the PedsQL 3.0 DM except for communication. Also, the regression analysis of this study showed that DKA was the independent influencing factor of the worry subscale.

Regarding the correlation between the number of severe hypoglycemic episodes and HRQOL, current results were in accord with some studies which hypothesized that the experience of suffering severe hypoglycemia could affect HRQOL for fear of their recurrence, such as the study was done by Nordfeldt and Ludvigsson 2005. Also, the study done by Abolfotouh et al. 2011 who conducted a quiz experimental study to assess the self-related quality of life and glycemic control in adolescents with type 1 diabetes in Alexandria, Egypt, they reported lower scores associated with an increasing number of hypoglycemic episodes. The previous studies came in line with the Canadian and Brazilian studies conducted by Harris et al. 2014 and Bahia et al. 2017, respectively. On the other hand, the study of Murillo et al. 2017b revealed that patients who had suffered significant hypoglycemia showed good scores in almost all dimensions of HRQOL, although these data were not valuable because of the small number (only 3 out of 136 patients) had been experiencing hypoglycemia.

\section{Conclusion}

The present study concluded that health-related quality of life (HRQOL) was similar in diabetic and non-diabetic children, while decreased in diabetic adolescents in relation to their healthy peers. It was negatively affected by the development of microvascular complications (especially diabetic neuropathy), while positively affected by achieving good metabolic control (HbA1c $<8 \%$ ).

\section{Abbreviations}

A.O.D: Age of onset of disease; D.D: Disease duration; DKA: Diabetic ketoacidosis; DM: PedsQL'M 3.0 Diabetes Module; DSPN: Distal symmetric polyneuropathy; GCS: PedsQL' ${ }^{\text {TM }} 4.0$ Generic Core Scales; HRQOL: Healthrelated quality of life; PedsQL ${ }^{\mathrm{T}}$. Pediatric Quality of Life Inventory ${ }^{\mathrm{T}}$,

T1DM: Type 1 diabetes mellitus; WHO: World Health Organization

\section{Acknowledgements}

We thank all children participated in the study and their families, nurses, and lab team for their participation and assistance.

\section{Authors' contributions}

All the authors have accepted responsibility for the entire content of this submitted manuscript and approved the submission. All authors have read the final manuscript, revised it, and agreed that the work is ready for publication.

Funding

By National Research Center.

\section{Availability of data and materials}

The datasets used and analyzed during the current study are available from the corresponding author on reasonable request.

\section{Ethics approval and consent to participate}

Approval was taken to conduct this research from the Ethical Committee of the faculty of Postgraduate Childhood Studies, Ain Shams University; the

Ethical Committee of the Faculty of Medicine, Ain Shams University; and the Ethical Committee of the National Research Centre (NRC).

Consent for publication

Not applicable

\section{Competing interests}

The authors declare that they have no competing interests.

\section{Author details}

${ }^{1}$ Department of Child Health, National Research Centre, Cairo, Egypt. 2Diabetes Clinics, Children's Hospital, Faculty of Medicine, Ain Shams University, Cairo, Egypt. ${ }^{3}$ Department of Medical Studies, Faculty of Postgraduate Childhood Studies, Ain Shams University, Cairo, Egypt. ${ }^{4}$ Department of Clinical Pathology, National Research Centre, Cairo, Egypt.

Received: 31 May 2019 Accepted: 8 August 2019

Published online: 05 September 2019

\section{References}

Abd-Elall BM, Mohamed MM, Hashim HA (2016) Health related quality of life of diabetic children and factors affecting it in Sohag University Hospital, Sohag, Egypt. J Med Sci Clin Res 4(7):11477-11485 49

Abdul-Rasoul M, AlOtaibi F, Abdulla A, Rahme Z, AlShawaf F (2013) Quality of life of children and adolescents with type 1 diabetes in Kuwait. Med Princ Pract 22(4):379-384

Abolfotouh MA, Kamal MM, El-Bourgy MD, Mohamed SG (2011) Quality of life and glycemic control in adolescents with type 1 diabetes and the impact of an education intervention. Int J Gen Med 4:141-152

Abuawad, M. S. (2013). Assessing quality of life of Palestinian diabetic patients; refugees and non-refugees: UNRWA and $\mathrm{MOH}$ health centers attendants (Master's thesis, Oslo and Akershus University College of Applied Sciences. Faculty of Social Sciences) 
Al-Akour N, Khader YS, Shatnawi NJ (2010) Quality of life and associated factors among Jordanian adolescents with type 1 diabetes mellitus. J Diabetes Complicat 24(1):43-47

AlBuhairan F, Nasim M, Al Otaibi A, Shaheen NA, Al Jaser S, Al Alwan I (2016) Health related quality of life and family impact of type 1 diabetes among adolescents in Saudi Arabia. Diabetes Res Clin Pract 114:173-179

Al-Hayek AA, Robert AA, Abbas HM, Itani MB, Al-Saeed AH, Juhani AE et al (2014) Assessment of health-related quality of life among adolescents with type 1 diabetes mellitus in Saudi Arabia. Saudi Med J 35(7):712-717

Alvarado-Martel D, Velasco R, Sánchez-Hernández RM, Carrillo A, Nóvoa FJ, Wägner AM (2015) Quality of life and type 1 diabetes: a study assessing patients' perceptions and self-management needs. Patient Prefer Adherence 9:1315-1323

American Diabetes Association (2018) Comprehensive medical evaluation and assessment of comorbidities: Standards of Medical Care in Diabetes-2018. Diabetes Care 41(Suppl. 1):S28-S37

Anderson BJ, Laffel LM, Domenger C, Danne T, Phillip M, Mazza C et al (2017) Factors associated with diabetes-specific health-related quality of life in youth with type 1 diabetes: the Global TEENs Study. Diabetes Care 40(8):1002-1009

Arabiat DH, Al Jabery MA (2013) Health related quality of life in paediatric chronic health conditions: a comparative study among children and adolescents in Jordan. Health 5(11):19

Bahia L, Kupfer R, Momesso D, Cabral DA, Tschiedel B, Puñales M et al (2017) Health-related quality of life and utility values associated to hypoglycemia in patients with type 1 diabetes mellitus treated in the Brazilian Public Health System: a multicenter study. Diabetol Metab Syndr 9(1):9

Bai JW, Lovblom LE, Cardinez M, Weisman A, Farooqi MA, Halpern EM et al (2017) Neuropathy and presence of emotional distress and depression in longstanding diabetes: results from the Canadian study of longevity in type 1 diabetes. J Diabetes Complicat 31(8):1318-1324

Barcellos SH, Carvalho LS, Lleras-Muney A (2014) Child gender and parental investments in India: are boys and girls treated differently? Am Econ J Appl Econ 6(1):157-189

Baş VN, Bideci A, Yeşilkaya E, Soysal AŞ, Çamurdan O, Cinaz P (2011) Evaluation of factors affecting quality of life in children with type 1 diabetes mellitus. J Diabetes Metab 2(8):154-158

Bisegger C, Cloetta B, von Bisegger U, Abel T, Ravens-Sieberer U (2005) Healthrelated quality of life: gender differences in childhood and adolescence. Soc Prev Med 50(5):281-291

Caferoğlu Z, Inanç N, Hatipoğlu N, Kurtoğlu S (2016) Health-related quality of life and metabolic control in children and adolescents with type 1 diabetes mellitus. J Clin Res Pediatr Endocrinol 8(1):67-73. https://doi.org/10.4274/ jcrpe.2051

Chawla $L$ (2004) The effects of political and economic transformations on children. In: Globalization and Children. Springer, Boston, pp 91-97

da Costa LMF, Vieira SE (2015) Quality of life of adolescents with type 1 diabetes. Clinics 70(3):173-179

De Boer IH, Rue TC, Cleary PA, Lachin JM, Molitch ME, Steffes MW et al (2011) Long-term renal outcomes of patients with type 1 diabetes mellitus and microalbuminuria: an analysis of the Diabetes Control and Complications Trial/Epidemiology of Diabetes Interventions and Complications cohort. Arch Intern Med 171(5):412-420

de Souza ACCB, Felício JS, Koury CC, Neto JFA, Miléo KB, Santos FM et al (2015) Health-related quality of life in people with type 1 diabetes mellitus: data from the Brazilian Type 1 Diabetes Study Group. Health Qual Life Outcomes 13(1):204. https://doi.org/10.1186/s12955-015-0396-0

Dobrota VD, Hrabac P, Skegro D, Smiljanic R, Dobrota S, Prkacin I et al (2014) The impact of neuropathic pain and other comorbidities on the quality of life in patients with diabetes. Health Qual Life Outcomes 12(1):171

El Wakeel MA, Abou-el-asrar M, El-kassas GM, Elabd MA, Zeid DA, Sabry RN, Awadallah E (2017) Urinary markers of oxidative DNA damage in type 1 diabetic children: relation to microvascular complications. Asian J Pharm Clin Res 10(10):318-322

El-Shakhs AE. (2013). A scale of socioeconomic status of the family. The scale manual. Cairo, Anglo-Egyptian Stationery

Fidler C, Elmelund Christensen T, Gillard S (2011) Hypoglycemia: an overview of fear of hypoglycemia, quality-of-life, and impact on costs. J Med Econ 14(5): 646-655

Floyd BD, Block JM, Buckingham BB, Ly T, Foster N, Wright R et al (2017) Stabilization of glycemic control and improved quality of life using a shared medical appointment model in adolescents with type 1 diabetes in suboptimal control. Pediatr Diabetes 18(3):204-212
Forsander G, Bøgelund M, Haas J, Samuelsson U (2017) Adolescent life with diabetes-gender matters for level of distress. Experiences from the national TODS study. Pediatr Diabetes 18(7):651-659

Frøisland DH, Graue M, Markestad T, Skrivarhaug T, Wentzel-Larsen T, DahlJørgensen K (2013) Health-related quality of life among Norwegian children and adolescents with type 1 diabetes on intensive insulin treatment: a population-based study. Acta Paediatr 102(9):889-895

Gadallah MA, Ismail TAAM, Aty NSA (2017) Health related quality of life among children with type I diabetes, Assiut city, Egypt. J Nurs Educ Pract 7(10):73-82

Gesuita R, Skrami E, Bonfanti R, Cipriano P, Ferrito L, Frongia P et al (2017) The role of socio-economic and clinical factors on $\mathrm{HbA} 1 \mathrm{c}$ in children and adolescents with type 1 diabetes: an Italian multicentre survey. Pediatr Diabetes 18(3):241-248

Gouveia MJ, Frontini R, Canavarro MC, Moreira H (2014) Quality of life and psychological functioning in pediatric obesity: the role of body image dissatisfaction between girls and boys of different ages. Qual Life Res 23(9): 2629-2638

Griffiths S, Murray SB, Bentley C, Gratwick-Sarll K, Harrison C, Mond JM (2017) Sex differences in quality of life impairment associated with body dissatisfaction in adolescents. J Adolesc Health 61(1):77-82

Gross JL, De Azevedo MJ, Silveiro SP, Canani LH, Caramori ML, Zelmanovitz T (2005) Diabetic nephropathy: diagnosis, prevention, and treatment. Diabetes Care 28(1):164-176

Hannula V, Hautala NM, Tossavainen P, Falck AA (2015) Social well-being of young adults with type 1 diabetes since childhood. The Oulu cohort study of diabetic retinopathy. Scand J Public Health 43(6):623-628

Harris S, Mamdani M, Galbo-Jørgensen CB, Bøgelund M, Gundgaard J, Groleau D (2014) The effect of hypoglycemia on health-related quality of life: Canadian results from a multinational time trade-off survey. Can J Diabetes 38(1):45-52

Hassan K, Loar R, Anderson BJ, Heptulla RA (2006) The role of socioeconomic status, depression, quality of life, and glycemic control in type 1 diabetes mellitus. J Pediatr 149(4):526-531

Hassan M, Musa N, Abdel Hai R, Fathy A, Ibrahim A (2017) Assessment of healthrelated quality of life in Egyptian adolescents with type 1 diabetes: DEMPU survey. J Pediatr Endocrinol Metab 30(3):277-283

Hilliard ME, Wu YP, Rausch J, Dolan LM, Hood KK (2013) Predictors of deteriorations in diabetes management and control in adolescents with type 1 diabetes. J Adolesc Health 52(1):28-34

Hirai FE, Tielsch JM, Klein BE, Klein R (2013) Ten-year change in self-rated quality of life in a type 1 diabetes population: Wisconsin Epidemiologic Study of Diabetic Retinopathy. Qual Life Res 22(6):1245-1253

Hoey H, Aanstoot HJ, Chiarelli F, Daneman D, Danne T, Dorchy H et al (2001) Good metabolic control is associated with better quality of life in 2,101 adolescents with type 1 diabetes. Diabetes Care 24(11):1923-1928

Ingerski LM, Laffel L, Drotar D, Repaske D, Hood KK (2010) Correlates of glycemic control and quality of life outcomes in adolescents with type 1 diabetes. Pediatr Diabetes 11(8):563-571

Jacobson AM, Braffett BH, Cleary PA, Gubitosi-Klug RA, Larkin ME, DCCT/EDIC research group (2013) The long-term effects of type 1 diabetes treatment and complications on health-related quality of life. Diabetes Care 36(10): $3131-3138$

Jafari P, Forouzandeh E, Bagheri Z, Karamizadeh Z, Shalileh K (2011) Health related quality of life of Iranian children with type 1 diabetes: reliability and validity of the Persian version of the PedsQL ${ }^{\mathrm{TM}}$ Generic Core Scales and Diabetes Module. Health Qual Life Outcomes 9(1):104

Jain V, Shivkumar S, Gupta O (2014) Health-related quality of life ( $\mathrm{Hr}$-Qol) in patients with type 2 diabetes mellitus. N Am J Med Sci 6(2):96

Jaser SS, Patel N, Xu M, Tamborlane WW, Grey M (2017) Stress and coping predicts adjustment and glycemic control in adolescents with type 1 diabetes. Ann Behav Med 51(1):30-38

Kleefstra N, Landman GW, Houweling ST, Ubink-Veltmaat L, Logtenberg SJ, Meyboom-de Jong B et al (2008) Prediction of mortality in type 2 diabetes from health-related quality of life (ZODIAC-4). Diabetes Care 31(5):932-933

Larsson HE, Vehik K, Haller MJ, Liu X, Akolkar B, Hagopian W et al (2016) Growth and risk for islet autoimmunity and progression to type 1 diabetes in early childhood: the environmental determinants of diabetes in the young study. Diabetes 65(7):1988-1995. https://doi.org/10.2337/db15-1180

Lawrence JM, Joyce P, Black MH, Anderson A, Hood K, Imperatore G et al (2012) Demographic and clinical correlates of diabetes-related quality of life among youth with type 1 diabetes. J Pediatr 161(2):201-207 
Lontchi-Yimagou E, Mapa-Tassou C, Dehayem MY, Essi MJ, Saji J, Takogue R et al (2017) The effect of free diabetes care on metabolic control and on healthrelated quality of life among youths with type 1 diabetes in Cameroon. BMJ Open Diabetes Res Care 5(1):e000397

Mahmood D, Singh BK, Akhtar M (2009) Diabetic neuropathy: therapies on the horizon. J Pharm Pharmacol 61(9):1137-1145

Murillo M, Bel J, Pérez J, Corripio R, Carreras G, Herrero X et al (2017a) Impact of monitoring health-related quality of life in clinical practice in children with type 1 diabetes mellitus. Qual Life Res 26(12):3267-3277

Murillo M, Bel J, Pérez J, Corripio R, Carreras G, Herrero X et al (2017b) Healthrelated quality of life (HRQOL) and its associated factors in children with type 1 diabetes mellitus (T1DM). BMC Pediatr 17(1):16

Nansel TR, Weisberg-Benchell J, Wysocki T, Laffel L, Anderson B, Steering Committee of the Family Management of Diabetes Study (2008) Quality of life in children with type 1 diabetes: a comparison of general and diabetesspecific measures and support for a unitary diabetes quality-of-life construct. Diabet Med 25(11):1316-1323

Naughton MJ, Ruggiero AM, Lawrence JM, Imperatore G, Klingensmith GJ, Waitzfelder B et al (2008) Health-related quality of life of children and adolescents with type 1 or type 2 diabetes mellitus: SEARCH for diabetes in youth study. Arch Pediatr Adolesc Med 162(7):649-657

Nielsen HB, Ovesen LL, Mortensen LH, Lau CJ, Joensen LE (2016) Type 1 diabetes, quality of life, occupational status and education level-a comparative population-based study. Diabetes Res Clin Pract 121:62-68

Nieuwesteeg A, Pouwer F, van der Kamp R, van Bakel H, Aanstoot HJ, Hartman E (2012) Quality of life of children with type 1 diabetes: a systematic review. Curr Diabetes Rev 8(6):434-443

Nordfeldt S, Ludvigsson J (2005) Fear and other disturbances of severe hypoglycaemia in children and adolescents with type 1 diabetes mellitus. J Pediatr Endocrinol Metab 18(1):83-92

Östberg V, Almquist YB, Folkesson L, Låftman SB, Modin B, Lindfors P (2015) The complexity of stress in mid-adolescent girls and boys. Child Indic Res 8(2): 403-423

Patterson CC, Gyürüs E, Rosenbauer J, Cinek O, Neu A, Schober E et al (2015) Seasonal variation in month of diagnosis in children with type 1 diabetes registered in 23 European centers during 1989-2008: little short-term influence of sunshine hours or average temperature. Pediatr Diabetes 16(8): 573-580

Paula JS, Braga LD, Moreira RO, Kupfer R (2017) Correlation between parameters of self-monitoring of blood glucose and the perception of health-related quality of life in patients with type 1 diabetes mellitus. Arch Endocrinol Metab 61(4):343-347

Ravens-Sieberer U, Erhart M, Wille N, Wetzel R, Nickel J, Bullinger M (2006) Generic health-related quality-of-life assessment in children and adolescents. Pharmacoeconomics 24(12):1199-1220

Rose AJ, Asher SR (2017) The social tasks of friendship: do boys and girls excel in different tasks? Child Dev Perspect 11(1):3-8

Sabry RN, El Wakeel MA, El-Kassas GM, Amer AF, El Batal WH, El-Zayat SR (2018) Serum Apelin: a new marker of early atherosclerosis in children with type 1 diabetes mellitus. Open Access Maced J Med Sci 6(4):613-617. https://doi. org/10.3889/oamjms.2018.144

Schwimmer JB, Burwinkle TM, Varni JW (2003) Health-related quality of life of severely obese children and adolescents. JAMA 289(14):1813-1819

Smith-Palmer J, Bae JP, Boye KS, Norrbacka K, Hunt B, Valentine WJ (2016) Evaluating health-related quality of life in type 1 diabetes: a systematic literature review of utilities for adults with

Stahl A, Straßburger K, Lange K, Bächle C, Holl RW, Giani G, Rosenbauer J (2012) Health-related quality of life among German youths with early-onset and long-duration type 1 diabetes. Diabetes Care 35:1736-1742 Diabetes Care, 35(11), 2412-2412

Tanna A, Sommaiya J, Dobariya J, Doshi P, Shah R (2016) Prevalence of dysmenorrhea and its effects on quality of life in college going girls. Int J Curr Adv Res 5(7):1093-1096

Tanner JM (1976) Clinical longitudinal standards for height, weight, height velocity, weight velocity, and stages of puberty. Arch Dis Child 51:170-179

Tesfaye S, Selvarajah D (2012) Advances in the epidemiology, pathogenesis and management of diabetic peripheral neuropathy. Diabetes Metab Res Rev 28(S1):8-14

Tofeeq R, Ahmed K, Saleem S (2017) Physical problems among children and adolescents complain of diabetes type I in Erbil City. Kufa J Nurs Sci 6(3):60مجل
Toffol E, Koponen P, Luoto R, Partonen T (2014) Pubertal timing, menstrual irregularity, and mental health: results of a population-based study. Arch Womens Ment Health 17(2):127-135

Van der Graaff J, Branje S, De Wied M, Hawk S, Van Lier P, Meeus W (2014) Perspective taking and empathic concern in adolescence: gender differences in developmental changes. Dev Psychol 50(3):881-888

Vanelli M, Chiarelli F, Chiari G, Tumini S (2003) Relationship between metabolic control and quality of life in adolescents with type 1 diabetes. Acta Biomed 1:13-17

Varni JW, Burwinkle TM, Jacobs JR, Gottschalk M, Kaufman F, Jones KL (2003) The PedsQL in type 1 and type 2 diabetes: reliability and validity of the pediatric quality of life inventory generic core scales and type 1 diabetes module. Diabetes Care 26(3):631-637

Varni JW, Seid M, Kurtin PS (2001) PedsQL ${ }^{\mathrm{TM}}$ 4.0: reliability and validity of the Pediatric Quality of Life Inventory ${ }^{\mathrm{TM}}$ Version 4.0 Generic Core Scales in healthy and patient populations. Med Care 39(8):800-812

Varni JW, Seid M, Rode CA (1999) The PedsQL ${ }^{\mathrm{T} M}$ : measurement model for the pediatric quality of life inventory. Med Care 37(2):126-139

Vileikyte L, Crews RT, Reeves ND (2017) Psychological and biomechanical aspects of patient adaptation to diabetic neuropathy and foot ulceration. Curr Diab Rep 17(11):109

Wang Z, Xie Z, Lu Q, Chang C, Zhou Z (2017) Beyond genetics: what causes type 1 diabetes. Clin Rev Allergy Immunol 52(2):273-286

Wiklund M, Malmgren-Olsson EB, Öhman A, Bergström E, Fjellman-Wiklund A (2012) Subjective health complaints in older adolescents are related to perceived stress, anxiety and gender-a cross-sectional school study in Northern Sweden. BMC Public Health 12(1):993

Williams J, Wake M, Hesketh K, Maher E, Waters E (2005) Health-related quality of life of overweight and obese children. JAMA 293(1):70-76

World Health Organization. (2011). WHO Anthro Plus for personal computers. Manual Software for assessing growth of the world's children and adolescents. (Version 3.2. 2, January 2011) and macros. Available on line at: http://www.who.int/childgrowth/software/en/

\section{Publisher's Note}

Springer Nature remains neutral with regard to jurisdictional claims in published maps and institutional affiliations.

\section{Submit your manuscript to a SpringerOpen ${ }^{\circ}$ journal and benefit from:}

- Convenient online submission

- Rigorous peer review

- Open access: articles freely available online

- High visibility within the field

- Retaining the copyright to your article

Submit your next manuscript at $>$ springeropen.com 